\title{
PERFORMANCE ASSESSMENT OF SEISMIC RETROFITTING MEASURES ON SILO STRUCTURES USING INNOVATIVE SEISMIC PROTECTION SYSTEMS
}

\author{
Marius Pinkawa $^{1}$, Benno Hoffmeister ${ }^{1}$, and Markus Feldmann ${ }^{1}$ \\ ${ }^{1}$ Institute of Steel Construction \\ Mies-van-der-Rohe-Straße 1, 52074 Aachen, Germany \\ e-mail: m.pinkawa@stb.rwth-aachen.de
}

Keywords: Industrial Structures, Silo Structures, Seismic Retrofitting, Seismic Protection Devices, Model Simplification, Incremental Dynamic Analyses

\begin{abstract}
This paper deals with a real-world silo structure and the assessment of its seismic performance and possible retrofitting measures by using seismic protection devices. Firstly, the silo structure is shortly described followed by a simplified numerical modelling approach with the aim to save computational effort as much as possible but still achieve a reliable response of demands and failure modes. A comparison between the complex and simplified model is done, showing a very good match in terms of its dynamic properties, which are Eigenfrequencies, modal shapes and effective modal masses. With this simplified model incremental dynamic analyses are conducted to assess the current performance of the structure as-built. Strengths and weaknesses are highlighted by the simulation outcomes. Following, two possible retrofitting strategies using torsion-based hysteretic dampers are discussed. Hereby it is shown, how the seismic device can be modelled in any finite element software by using rheological models. In one strategy of seismic retrofit an innovative torsional device is inserted at single bays of the structural frame. In the second strategy the steel torsional hysteretic damper is applied at the base of the steel columns, such that the structure is separated from the ground. The first retrofit approach proves to be effective in prohibiting a severe sides-way collapse which occurred for some earthquakes. The second shows to be greatly effective in preventing failure and also decreases deformation demands on the structure efficiently.
\end{abstract}




\section{INTRODUCTION}

Seismic events may have disastrous effects on industrial sites; for example the Duzce earthquake in Turkey 1999, the L'Aquilla earthquake 2009 in Italy or the more recent Tohoku earthquake in Japan 2011 caused huge financial losses, as they all affected important industrial areas. One reason for the high losses are the high financial investment costs gathered on industrial sites which are at risk due to direct damage of industrial components and facilities. However, also more frequent smaller seismic events can lead to impairment of the functionality of key components and thus cause costly business interruptions. A further special characteristic of industrial facilities is the usual high risk potential of environmental damage as well as potentially threats to health and life of employees. For these reasons industrial structures are ideally suited for seismic design and retrofit by using special seismic protection systems.

The European project "PROINDUSTRY - Seismic PROtection of INDUSTRial plants by enhanced steel based sYstems" aims at developing such enhanced seismic protection devices applicable to new and already existing industrial structures. One of the several case studies investigated in the scope of the project is a large silo structure located in a steel plant. This silo is a very important member of the supply chain and its loss of functionality may lead to business disruption of the whole plant.

In this paper this realistic silo structure is firstly investigated as-built and further on different seismic retrofit strategies are applied. To assess on the effectiveness of the seismic retrofitting measure incremental dynamic analyses are the most detailed tools available. Due to the scatter in the characteristics of natural earthquakes a big amount of analyses needs to be conducted to obtain reliable results. As especially industrial facilities are often large and of complex nature, computational effort might be unmanageable. Therefore, usually a simplified model of the complex structure is sought after, which preserves characteristic dynamic behavior by being feasible to handle. Moreover, in order to obtain a reliable structural response not only the structure itself needs to be modelled realistically, also the applied seismic device needs to be represented appropriately. If the used finite element software does not provide special elements to model the behavior, which is often the case for new innovative seismic devices, it will be needed to use more or less complex rheological models or to implement ones own algorithm in the analysis core of the used software.

After a short description of the case study, a simplified modelling approach for the complex structure as well as the seismic device is presented. With these models first the as-built seismic performance is assessed and secondly two retrofitting strategies are investigated and its outcomes are compared to the initial stage.

\section{SILO STRUCTURE CASE STUDY}

\subsection{Description of the case study}

The case study investigated herein is an elevated silo structure with several rectangular silos constructed in the early 70s. The silo is part of a huge steel plant and stores iron ore, limestone and sinter. Thus, the structure has a key role in the storage and supply chain of the whole industrial facility. When the silo is out of function, this will lead to a costly downtime of several other operations within the industrial site. An assessment of the seismic performance and a potential seismic upgrade is therefore highly advantageous. 

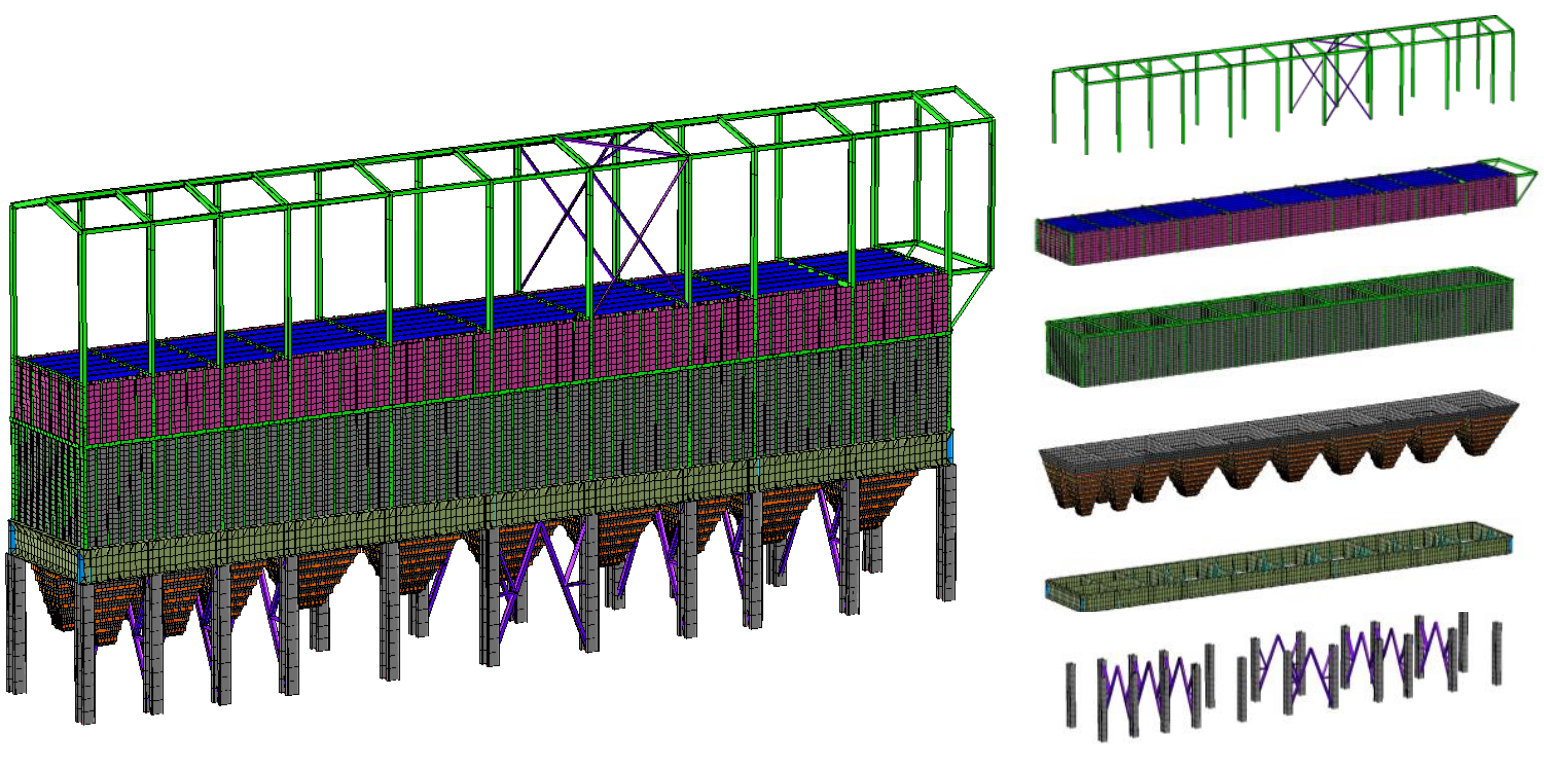

Figure 1: Silo steel structure. Complete model (left) and single subassemblies of silo structure (right).

The building has dimensions of $\mathrm{W} \times \mathrm{H} \times \mathrm{D}$ equal to $78 \mathrm{x} 40 \mathrm{x} 11 \mathrm{~m}$. The structural layout and main characteristics can be seen in Figure 1. The most significant single subassemblies of the structure are shown on the right of Figure 1. The top is made of a roof beam structure, which is based on vertically stiffened shells and horizontal beams. The level below is formed by the silo shaft composed of shells with vertical stiffeners and an upper ring beam. Adjoining are the silo hopper shells stiffened by horizontal beams. Below is the hopper skirt support, which is also strengthened with vertical stiffener plates. Finally, at the lowest level the steel supporting structure is visible, which is made of heavy built-up columns and braced frames, whereby inverted V-bracings are used. It can be seen that 6 braces are built in the short Y-direction which will be named transversal direction in the following, and 2 braces in the long X-direction, labeled as longitudinal direction. Probably, the unbalanced number of braces in the two directions is attributed to the fact that wind forces were the essential considerations for the design of the bracing system, thus more braces are needed in transversal direction due to the big area where wind forces can act on. The calculated mass of stored material equals approximately 25,000 $\mathrm{t}$ and the self-weight of the steel structure equals to 2,400 t. The structure is made of S275 structural steel.

\subsection{Description of the simplified model}

The complete finite element model shown in Figure 1 is very complex and has a large number of degrees of freedom. The total number of dynamic simulations equals to 198, which results from two sets of ground motions (low-to-medium and high seismicity) with each eleven ground motion time histories times nine scaling factors. Taking into account that both the original and the retrofitted have to be simulated the number already rises to 396 dynamic time history simulations. Moreover this number is the absolute minimum. It does not consider repeated simulations due to different retrofitting strategies and varying parameters of the used seismic protection device and does not take into account several runs due to debugging . Thus, in total several hundred analyses will be needed to be conducted. Not only the computational time, but also the limited storage for simulation output may show up to be problematic. Therefore, due 
to the huge amount of dynamic simulations which need to be conducted during the PROINDUSTRY project a computationally more efficient model was sought after.

The approach in simplifying the numerical model was to concentrate on the supporting structure and neglect the silo structure. This assumption was based on several reasons: Firstly, the silo shells are quite thick and massively stiffened, such that global failure of the structure is assumed to take place in the steel supporting structure rather than in the steel silos. Secondly, the dynamic modelling of the bulk material inside the silo would be needed to obtain reliably local failure of the silo shell. However, complex interaction models would be needed which further need several input parameters afflicted with a high level of uncertainties. Thus, on the one hand the reliability of the sophisticated approach would be still questionable and on the other hand the complexity excludes practically the simulation of several hundreds of analyses.

However, with the simplified approach just demands on the supporting structure and the mass points representing the silos are determined, without giving information about demands on the silo shells. However, peak forces and differential displacements obtained by the simplified model might be statically applied to the bottom or center of gravity of the silos bins. In a more complex approach, for bypassing this lack of information acceleration time history of the supporting structure may be applied to the bottom of the silo structure in a separate analysis, i.e. a cascaded approach similar to the floor response spectrum method used for SDOF systems. In this case the most detrimental excitation cases could be applied, to avoid extensive simulations but still to obtain reliable performance of the superstructure.

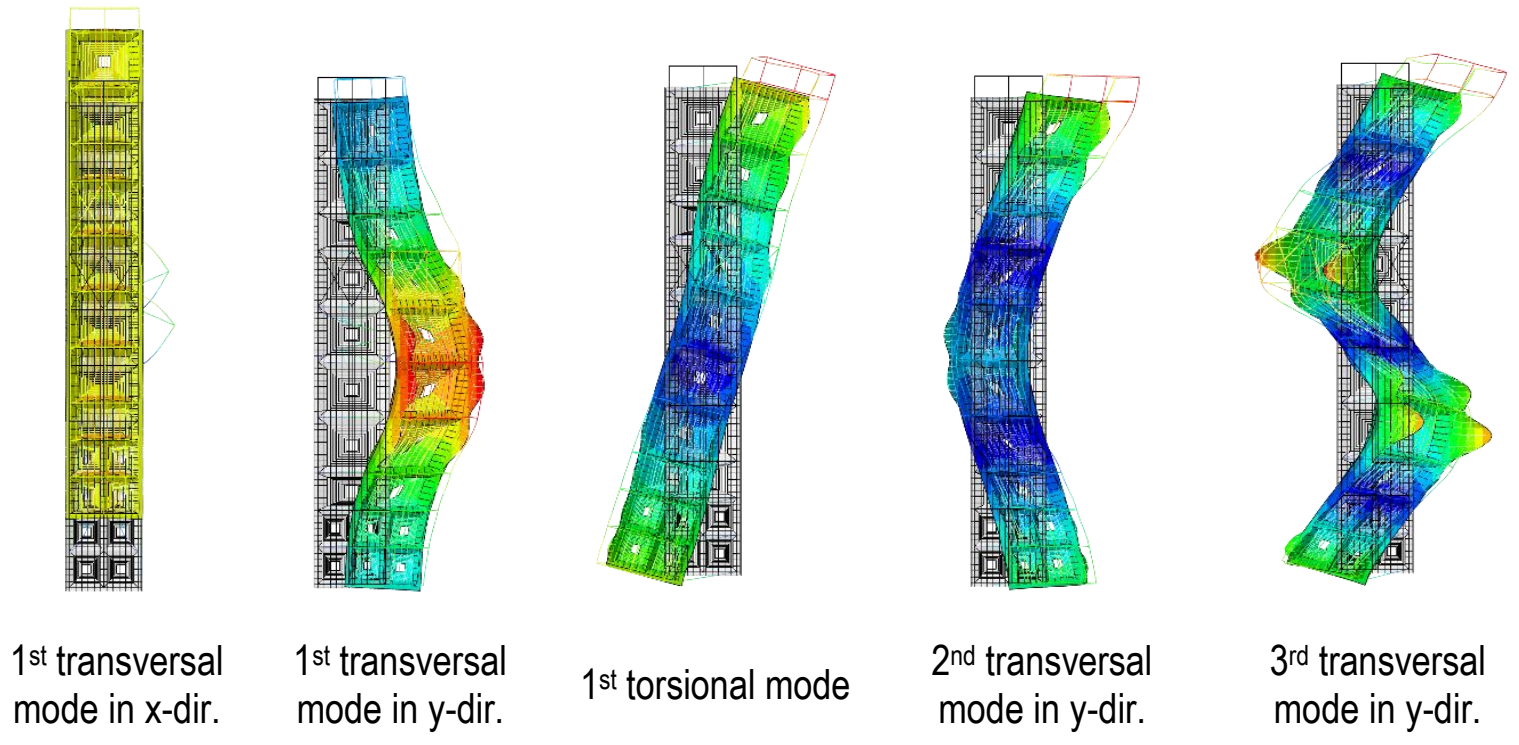

Figure 2: First five global mode shapes of complex structure.

Summarizing a simplified model is sought after, where the supporting structure is modelled explicitly by beam elements and the superstructure is taken into account by equivalent mass and stiffness properties applied to the supporting structure. Considering the Eigenmodes of the complex model shown in Figure 2, it is obvious that the superstructure cannot be modelled as rigid. Thus, a simplified model needs to capture the flexible behavior of the superstructure without explicitly modelling it. The flexibility needs to be modelled at the connection of the columns to the silos, representing the joint details shown in Figure 4 left. Finally, the simplified model shown in Figure 3 has been found to be appropriate. The silos are represented by mass points, which are located at the center of gravity of the full silo bins. A configuration of all silo bins totally filled has been investigated in this research work, which is in general the most critical 
situation. Torsional response due to extreme misbalanced filling configurations might be also critical, however this issue was not investigated further herein. The silo mass points are connected to each other by several constraints. The cross sections are modelled as fibre elements, where the stress strain law is intergrated at every interpolation point. Conservatively, an almost elastic - ideal plastic stress strain curve with a yield point of $27.5 \mathrm{kN} / \mathrm{cm}^{2}$ has been assumed. The connection of the silo mass assembly with the column tops was the key point and is discussed in the following.

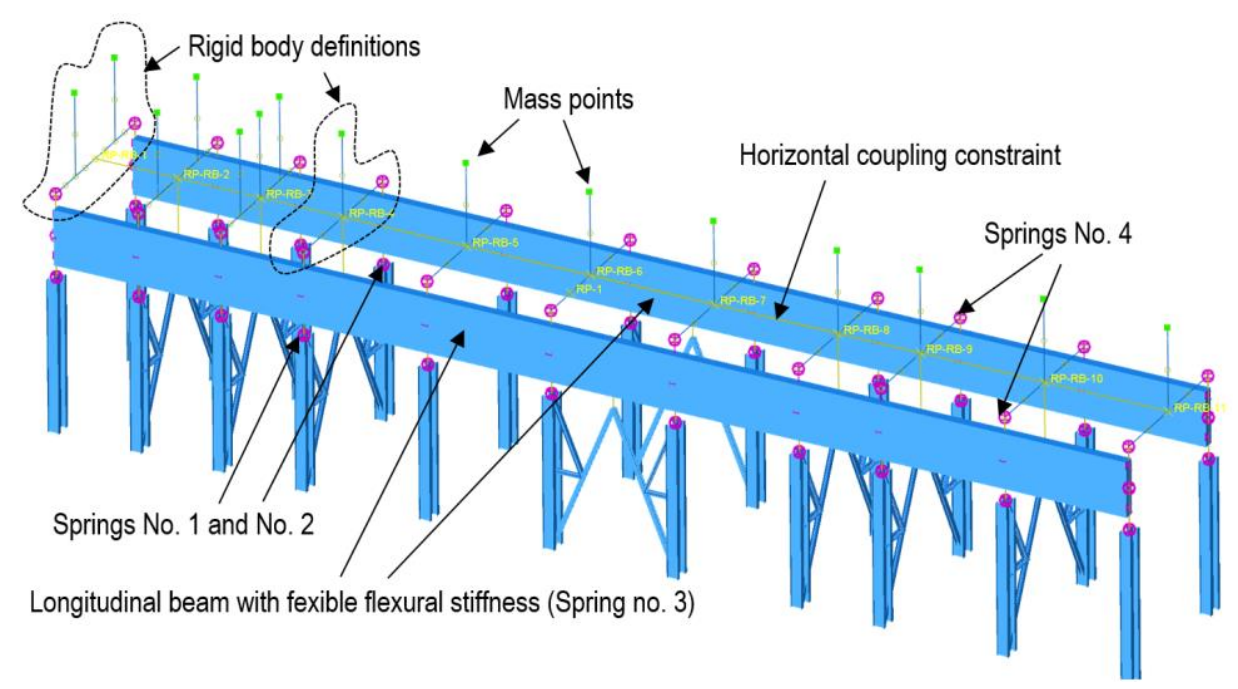

Figure 3: Simplified model with explicitly modelled supporting structure made of columns and frame braces with equivalent springs and couplings in order to take the flexible superstructure into account.

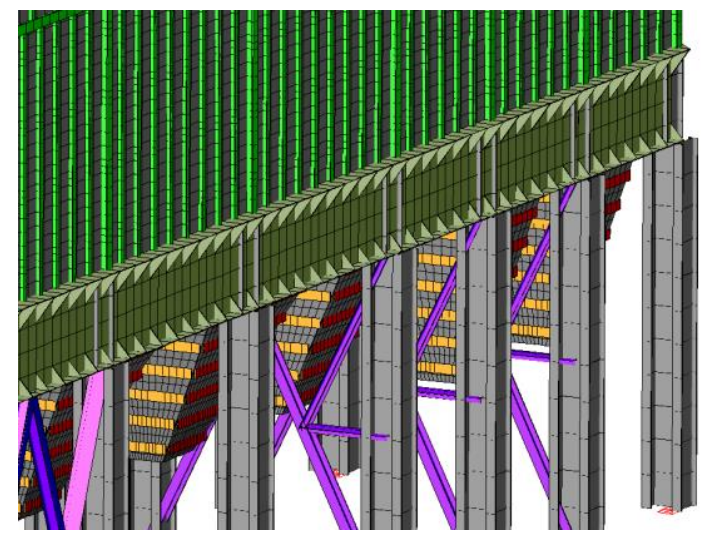

Figure 4: Connection detail between supporting and superstructure at columns.

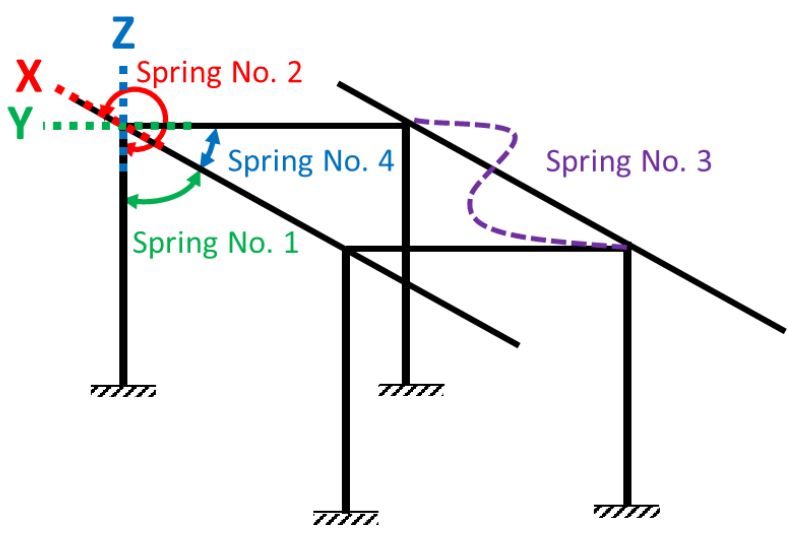

Figure 5: Schematical drawing of spring subassembly.

In order to obtain a reliable response of the simplified model, in total four spring or flexural stiffness properties respectively have been applied to the model. A schematical drawing of these springs can be found in Figure 5. A short description of each spring characteristic is given in the following Table 1 .

The spring parameters were calibrated by a trial-and-error procedure until three aspects in the simplified model matched the complex model's ones for the relevant modes:

- Eigenfrequencies

- Modal shapes

- Effective modal masses 


\section{Spring No. 1}

- Rotational spring about global Y-axis

- Connecting column top nodes and node of longitudinal beam

- Dominant spring for fundamental mode, not affecting higher relevant modes

\section{Spring No. 2}

- Rotational spring about global X-axis

- Connecting column top nodes and node of longitudinal beam

- Influencing higher relevant modes

\section{Spring No. 3}

- Rather a smeared stiffness property than a spring

- Flexural stiffness about local z-Axis of longitudinal beams (i.e. also global Z-Axis)

- Influencing higher modes, is needed to achieve transversal flexible behavior

\section{Spring No. 4}

- Rotational spring about global Z-axis

- Connecting longitudinal beam and transversal rigid T-type beam nodes. Influencing higher modes, is needed for the transversal flexible behavior to distribute transversal deflection from the higher displacement value at the middle to both ends in the longitudinal dimension, or in other words to form a more smooth transition from the middle bulge to the outer frames (see e.g. Figure 2: 1st transversal mode in y-dir.)

Table 1: Spring characteristics used in simplified beam model.

The final spring values which yielded the best match of the dynamic properties are given in Table 2. Whereby the rotational springs No. 1, 2 and 4 can be specified directly, the flexural stiffness of the longitudinal beams (spring No. 3) is achieved by defining a cross section with dimensions of $70 \times 400 \mathrm{~cm}$ and an elastic modulus of $21000 \mathrm{kN} / \mathrm{cm}^{2}$.

\begin{tabular}{ccrc}
\hline Spring No. & Label & \multicolumn{1}{c}{ Value } & Units \\
\hline 1 & $\mathrm{~K}_{1, \phi}$ & 610000 & $\mathrm{kNm} / \mathrm{rad}$ \\
2 & $\mathrm{~K}_{2, \phi}$ & 5500 & $\mathrm{kNm} / \mathrm{rad}$ \\
3 & $\mathrm{~K}_{3, \mathrm{EI}}$ & 24010000 & $\mathrm{kNm}$ \\
4 & $\mathrm{~K}_{4, \phi}$ & 10000000 & $\mathrm{kNm} / \mathrm{rad}$ \\
\hline
\end{tabular}

Table 2: Spring parameters used for the simplified model.

In the following the achieved match of dynamic properties between the simplified beam model and the full structure model is shown. Modal frequencies of the first five modes are shown in Table 3. Effective modal masses in both horiziontal directions are listed in Table 4. The total mass of the system is $27400 \mathrm{t}$. Corresponding modal shapes of the simplified and the full complex model are shown in Figure 6 to Figure 10 for comparison. It can be stated that for the most relevant modes no. 1, 2 and 4, the dynamic properties of the complex model are well represented with the simplified beam model. 


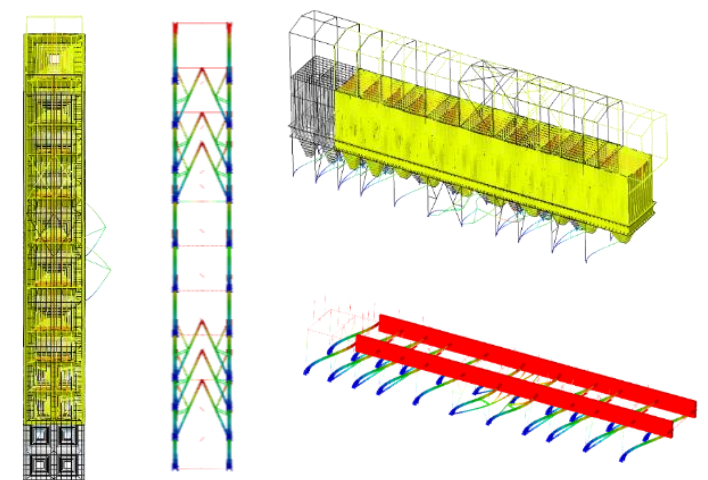

Figure 6: 1st global transversal mode (Mode 1).
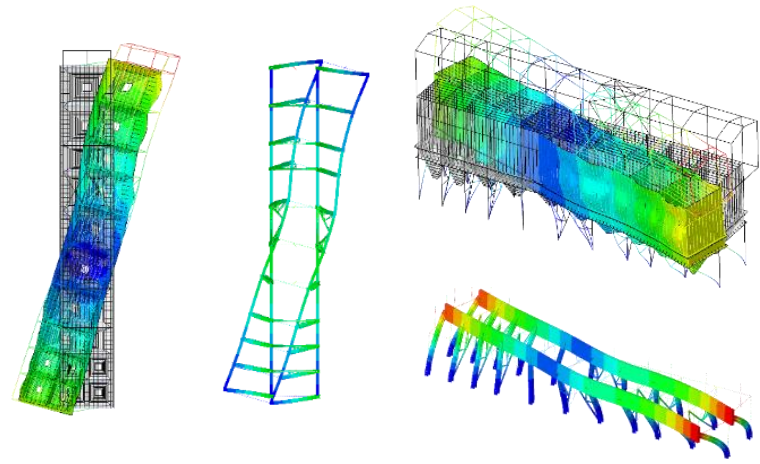

Figure 8: 1st global torsional mode (Mode 3).
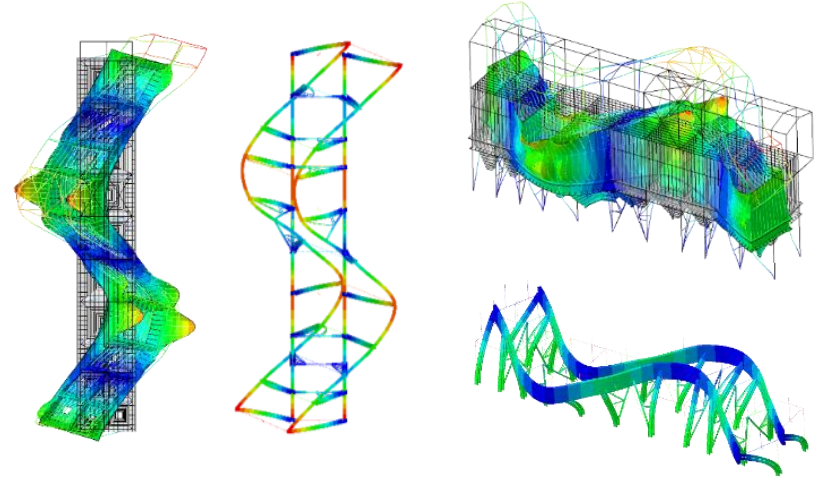

Figure 10: 3rd global transversal mode (Mode 5).
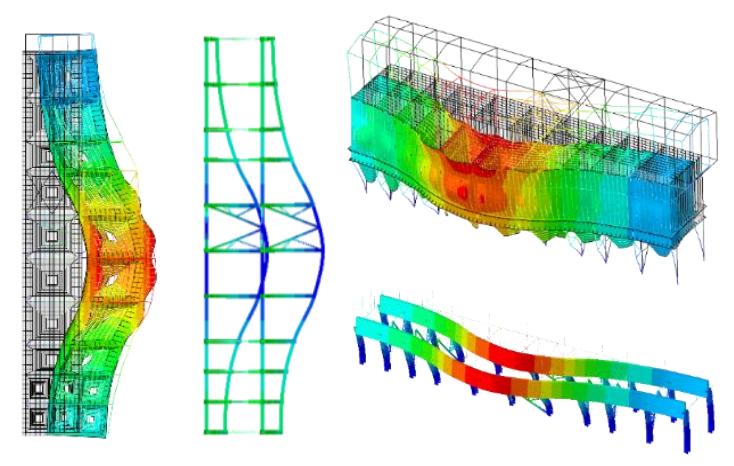

Figure 7: 1st global transversal mode (Mode 2).
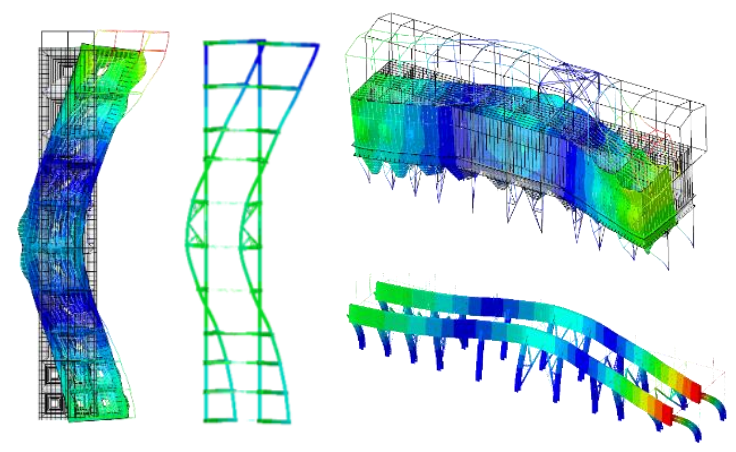

Figure 9: 2nd global transversal mode (Mode 4).

\begin{tabular}{|c|c|c|c|}
\hline \multirow{2}{*}{$\begin{array}{l}\text { Mode } \\
\text { No. }\end{array}$} & \multicolumn{2}{|c|}{ Modal period [s] } & \multirow{2}{*}{$\begin{array}{c}\text { Ratio } \\
\text { simplified } \\
\text { complex }\end{array}$} \\
\hline & $\begin{array}{c}\text { Complex } \\
\text { model }\end{array}$ & $\begin{array}{c}\text { Simplified } \\
\text { model }\end{array}$ & \\
\hline 1 & 1.69 & 1.69 & $100 \%$ \\
\hline 2 & 1.24 & 1.24 & $100 \%$ \\
\hline 3 & 0.94 & 0.90 & $95 \%$ \\
\hline 4 & 0.76 & 0.72 & $95 \%$ \\
\hline 5 & 0.62 & 0.59 & $94 \%$ \\
\hline
\end{tabular}

Table 3: Comparison of modal periods of complex and simplified model.

\begin{tabular}{|c|c|c|c|c|c|c|c|}
\hline \multirow{2}{*}{$\begin{array}{l}\text { Mode } \\
\text { No. }\end{array}$} & \multicolumn{2}{|c|}{$\begin{array}{l}\text { Effective modal mass }[\mathrm{t}] \\
\text { Longitudinal direction }\end{array}$} & \multirow{2}{*}{$\begin{array}{c}\text { Ratio } \\
\text { simpl. / } \\
\text { complex }\end{array}$} & \multirow{2}{*}{$\begin{array}{l}\text { Mode } \\
\text { No. }\end{array}$} & \multicolumn{2}{|c|}{$\begin{array}{l}\text { Effective modal mass }[\mathrm{t}] \\
\text { Transversal direction }\end{array}$} & \multirow{2}{*}{$\begin{array}{l}\text { Ratio } \\
\text { simpl. / } \\
\text { complex }\end{array}$} \\
\hline & $\begin{array}{l}\text { Complex } \\
\text { model }\end{array}$ & $\begin{array}{l}\text { Simplified } \\
\text { model }\end{array}$ & & & $\begin{array}{l}\text { Complex } \\
\text { model }\end{array}$ & $\begin{array}{l}\text { Simplified } \\
\text { model }\end{array}$ & \\
\hline 1 & 27299 & 27285 & $100 \%$ & 1 & 0 & 0 & - \\
\hline 2 & 0 & 0 & - & 2 & 22661 & 22311 & $98 \%$ \\
\hline 3 & 0 & 0 & - & 3 & 3 & 4 & $133 \%$ \\
\hline 4 & 0 & 0 & - & 4 & 4345 & 4630 & $107 \%$ \\
\hline 5 & 0 & 0 & - & 5 & 72 & 185 & $257 \%$ \\
\hline
\end{tabular}

Table 4: Comparison of effective modal masses in longitudinal (left) and transversal (right) direction. 


\subsection{Seismic input}

As input for the Incremental Dynamic Analyses recorded ground motion time histories have been used. A detailed description on the selection criteria and characteristics of the accelerograms can be found in [1]. Two sets with each eleven ground motions have been assembled: One set representative for low-medium seismicity regions and one representative for high seismicity regions (see Table 5 and 6 respectively). For the matching of the target spectrum the geometrical mean of both horizontal components has been used. Figure 1 and Figure 12 show the individual spectra of the selected accelerograms along with the mean and target spectrum. All three components - two horizontal and one vertical - were taken from the same recording. Also the vertical component has been applied in the simulations, because it might be critical due to the very high gravity loads present in the silo structures.

For the incremental dynamic analyses in total nine scale factors - different ones for the lowmedium seismicity and high seismicity ground motion sets - have been used. The scale factors were chosen such that different return periods have been covered, starting from rather frequent earthquakes (return period of 60 years) to extreme rare earthquakes (return period of 2475 years).

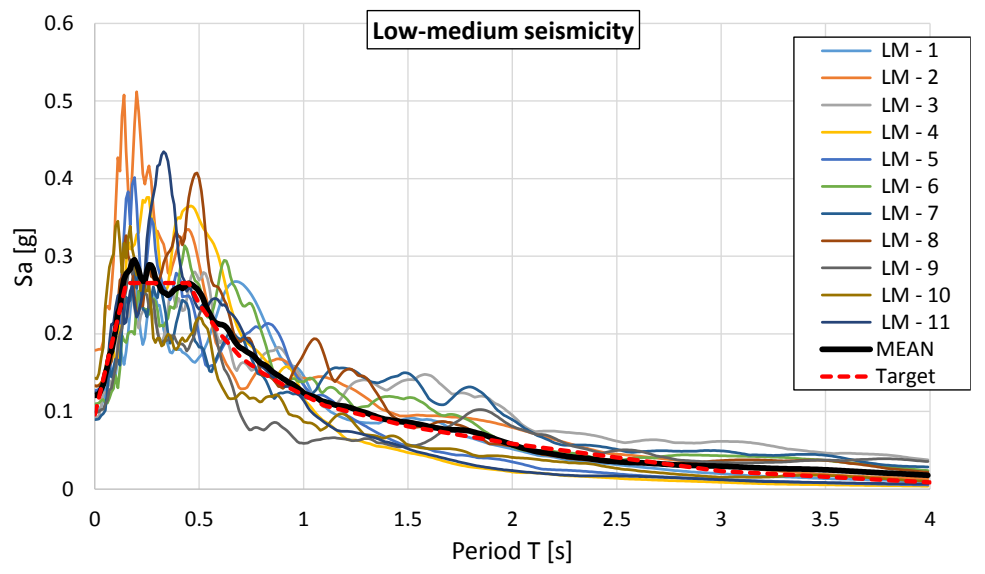

Figure 11: Low-medium seismicity acceleration spectra

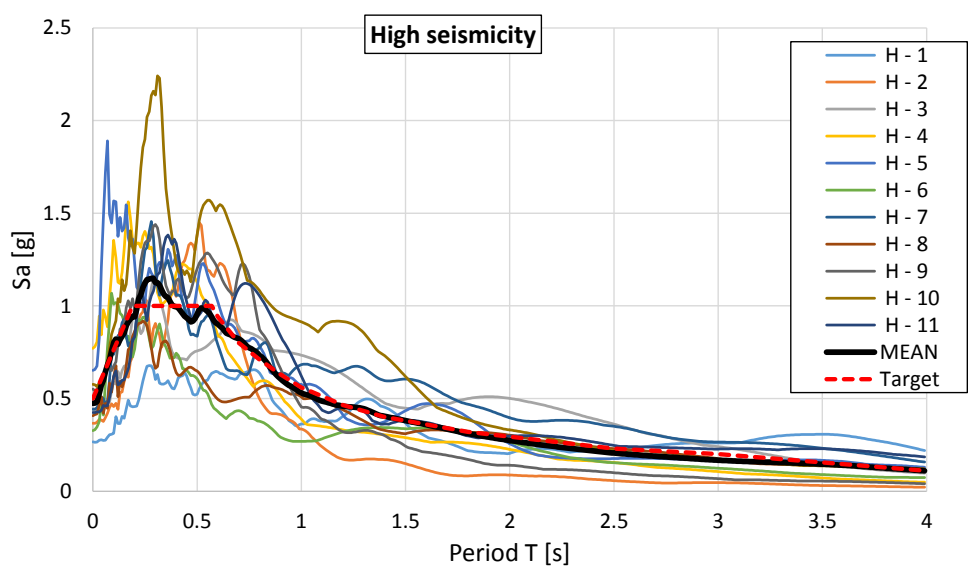

Figure 12: High seismicity acceleration spectra

\begin{tabular}{cccc}
\hline Label & Event location & $\mathrm{M}_{\mathrm{W}}$ & $\begin{array}{c}\mathrm{R} \\
{[\mathrm{km}]}\end{array}$ \\
\hline LM - 1 & Friuli & 6 & 14 \\
LM - & Friuli & 6 & 9 \\
LM - 3 & Lazio Abruzzo & 5.9 & 16 \\
LM - 4 & Umbria Marche & 6 & 22 \\
LM - 5 & Tabas & 7.3 & 68 \\
LM - 6 & Manjil & 7.4 & 81 \\
LM - 7 & Komilion & 5.4 & 15 \\
LM - 8 & Izmit & 7.6 & 96 \\
LM - 9 & Izmit & 5.8 & 25 \\
LM - 10 & Emilia & 6 & 19 \\
LM - 11 & Christchurch & 5.3 & 17 \\
\hline \multicolumn{4}{c}{}
\end{tabular}

Table 5: Low-medium seismicity set

\begin{tabular}{cccc}
\hline Label & Event location & $\mathrm{M}_{\mathrm{W}}$ & $\begin{array}{c}\mathrm{R} \\
{[\mathrm{km}]}\end{array}$ \\
\hline $\mathrm{H}-1$ & South Iceland & 6.4 & 5 \\
$\mathrm{H}-2$ & Montenegro & 6.9 & 25 \\
$\mathrm{H}-3$ & Erzincan & 6.6 & 13 \\
$\mathrm{H}-4$ & Gazli & 6.7 & 11 \\
$\mathrm{H}-5$ & Izmit & 7.6 & 20 \\
$\mathrm{H}-6$ & South Iceland & 6.5 & 5 \\
$\mathrm{H}-7$ & Duzce & 7.1 & 5 \\
$\mathrm{H}-8$ & Darfield & 7.1 & 18 \\
$\mathrm{H}-9$ & Imperial Valley & 6.5 & 27 \\
$\mathrm{H}-10$ & Loma Prieta & 6.9 & 7 \\
$\mathrm{H}-11$ & Northridge & 6.7 & 20 \\
\hline
\end{tabular}

Table 6: High seismicity set 


\section{SEISMIC PERFORMANCE OF AS-BUILT STRUCTURE}

First the as-built structure has been subjected to all earthquake time histories and scale factors. The most important outcomes are discussed in the following sections separated for the low-medium and the high seismicity ground motion sets. In general all simulations have been conducted geometrically fully nonlinear (large displacement theory).

\subsection{Low-medium seismicity ground motion set}

The incremental dynamic analyses with the low-medium seismicity accelerograms showed no larger convergence problems. Figure 14 shows the displacement at the top of the supporting structure in longitudinal and transversal direction as function of peak ground acceleration PGA. At the lowest scale factors the structure behaved almost elastic. For lower scale factors braces buckled in compression and yielded in tension. For the highest scale factors strong yielding and buckling occurred in almost all braces. Also all columns yielded at their base. However, no failure of the structure occurred for all of the earthquakes even at the highest scale factor.
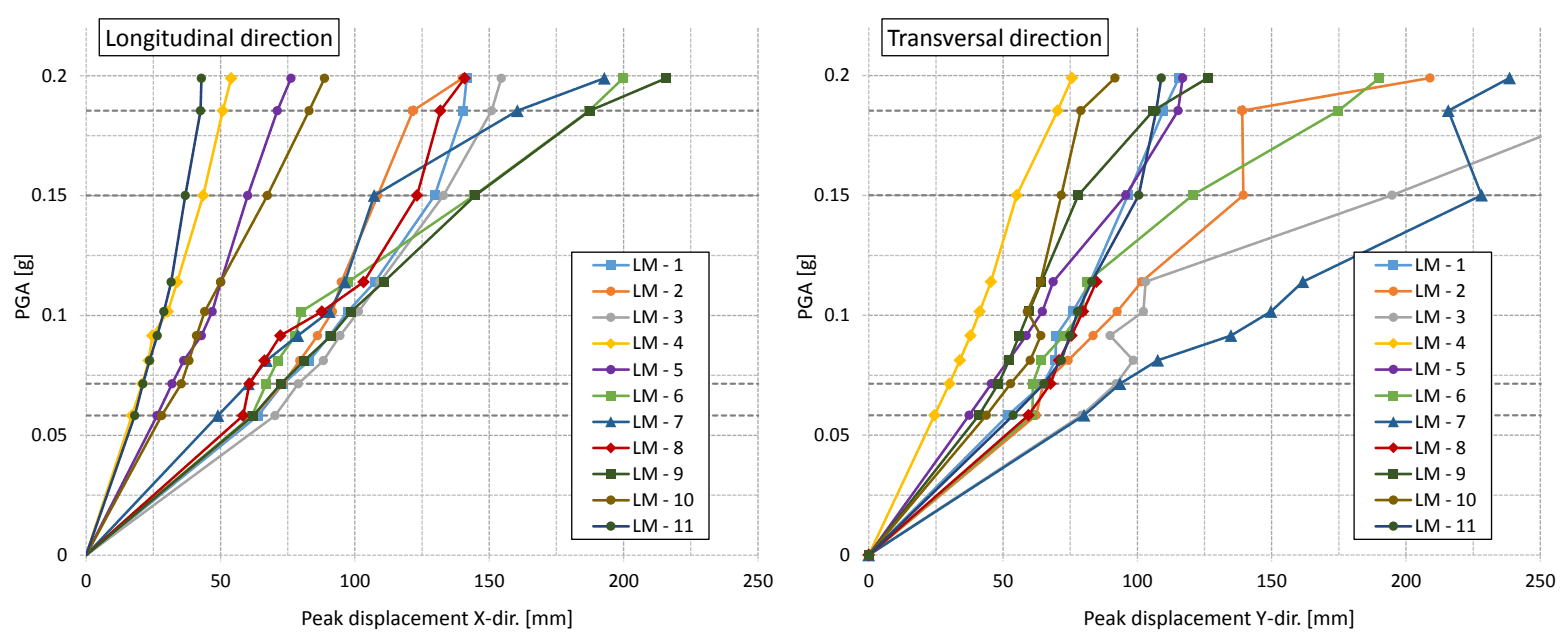

Figure 13: Low-medium seismicity IDA curves in longitudinal X- (left) and transversal Y-direction (right)

\subsection{High seismicity ground motion set}

As expected the high seismicity ground motion set provided much higher demands on the structure. Strong yielding patterns (yielding and buckling of braced frames, yielding of columns at base and top) occurred already for low scale factors. Figure 14 shows the corresponding IDA curves. At higher scale factors convergence difficulties occurred, thus, the IDA curves are not complete, as just fully completed simulations are shown in the diagram. The premature abortion of simulations mainly arrived due to collapse of the structure or excessive deformations of braces. The performance of the structure for each earthquake is summarized in Table 7. 

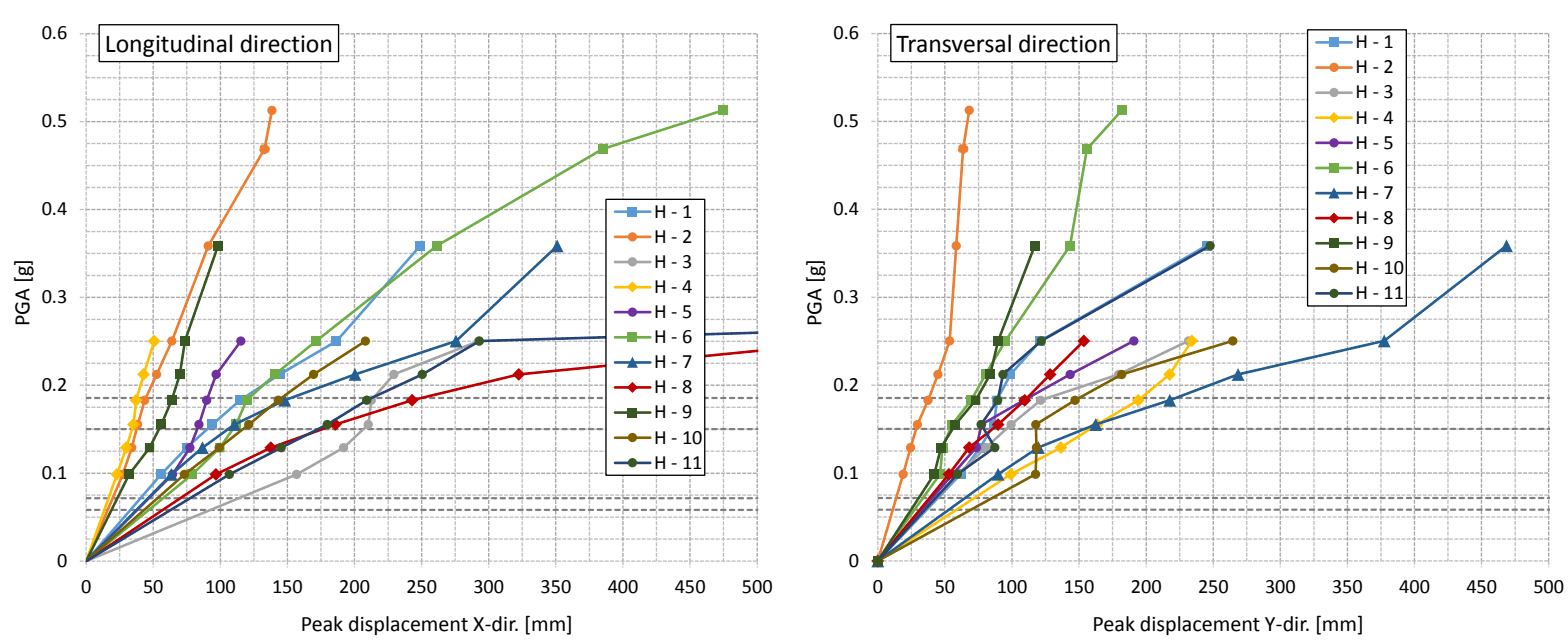

Figure 14: High seismicity IDA curves in longitudinal X- (left) and transversal Y-direction (right).

\begin{tabular}{cl}
\hline Ground motion & Comment \\
\hline $\mathrm{H}-1$ & Excessive deformation of one brace causes convergence problems \\
$\mathrm{H}-2$ & Full range of scale factors finished \\
$\mathrm{H}-3$ & Global sidesway collapse in longitudinal direction \\
$\mathrm{H}-4$ & Five braces exhibit excessive deformations leading to abortion of the simulation \\
$\mathrm{H}-5$ & Excessive deformation of one brace causes convergence problems \\
$\mathrm{H}-6$ & Full range of scale factors finished \\
$\mathrm{H}-7$ & Three braces deform extensively and cause abortion of the simulation \\
$\mathrm{H}-8$ & Global sidesway collapse in longitudinal direction \\
$\mathrm{H}-9$ & Five braces exhibit excessive deformations leading to abortion of the simulation \\
$\mathrm{H}-10$ & Three braces deform extensively and cause abortion of the simulation \\
$\mathrm{H}-11$ & Global sidesway collapse in longitudinal direction \\
\hline
\end{tabular}

Table 7: Comments on performance of IDA simulations.

Three of eleven earthquakes triggered a clear sides-way collapse in longitudinal direction. This highlights one of the weakness of the structural layout. Whereby in the transversal direction the structure is stiffened by in total 6 braced frames just two braced frames exist in the longitudinal direction. Obviously wind forces have governed the brace layout, where due to a much larger influence area the resulting lateral force is high in transversal direction and is relatively low in longitudinal direction. The evolution of the typical sides-way collapse is illustrated in Figure 15. Yielding at the bottom of columns is followed by yielding and buckling of the two frame braces and yielding of column tops and finally the structure collapses due to PDelta-effects. In six of the eleven earthquakes the simulation aborted prematurely either due to torsional buckling of the columns, which was triggered by out of plane displacements of the bracing frames, or due to or excessive deformations of the braces. See Figure 16 for an impression. Two of the eleven earthquakes finished without signs of global collapse. 


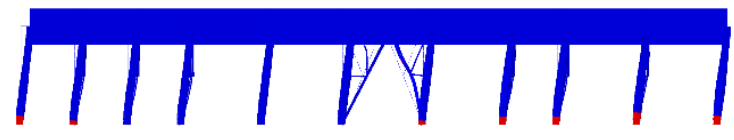

$8.98 \mathrm{~s}$ - Yielding of some column foots

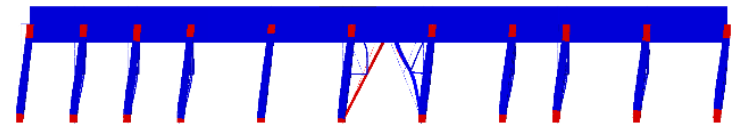

$9.00 \mathrm{~s}-$ Yielding at all column foots and tops

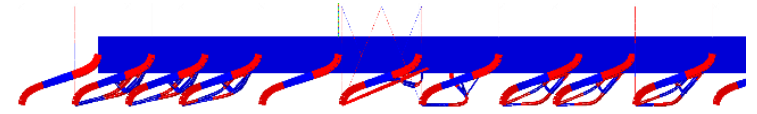

13.77 s - Sides-way collapse of structure

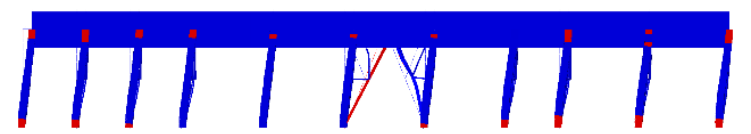

$8.99 \mathrm{~s}$ - Additional yielding of column tops and brace

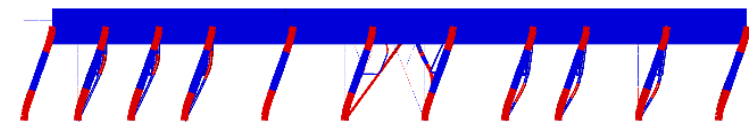

$12.00 \mathrm{~s}$ - Strong yielding of all columns and braces

Figure 15: Chronological evolution of sides-way collapse which occurred for earthquakes no. 3,8 and 11 , deformation scale factor 1.

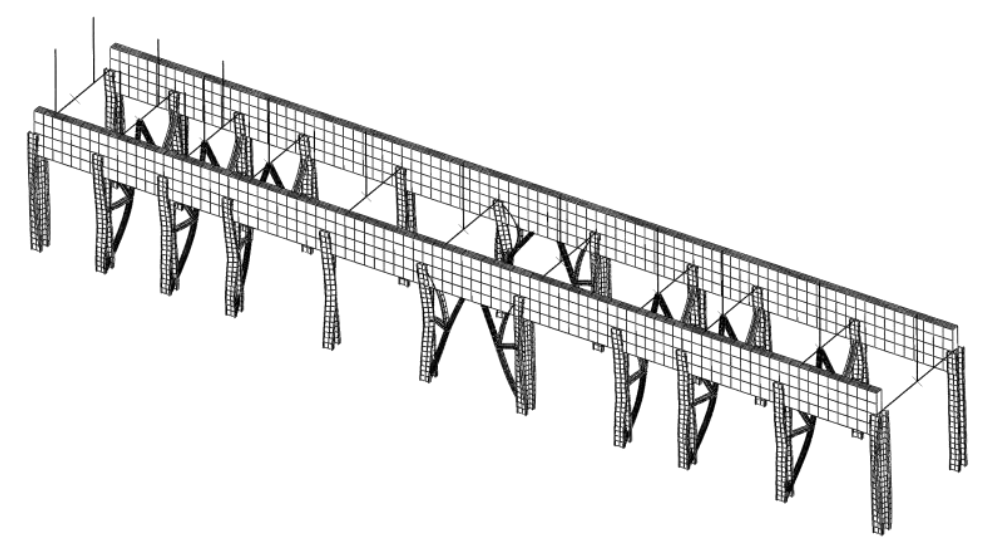

Figure 16: Failure mode torsional buckling of columns triggered by out of plane displacement of brace layer, deformation scale factor 1 .

\section{SEISMIC RETROFITTING STRATEGIES}

The principle of torsional yielding of steel rods shall be used in the phenomenologically modelled applied seismic devices. Such devices exist for example as Multi-Directional Hysteretic Devices explained in [2]. After a short introduction into the hysteretic behavior of such devices two generic retrofitting strategies will be investigated: As first retrofitting strategy a torsional damping device is applied inside the braced frames and as second strategy it is used as base isolation layer inserted between ground and structure at each column base.

\subsection{Torsional energy dissipation devices}

The seismic device hysteresis loop is shown in Figure 17. The most important aspects are the beneficial stiffening effect for higher displacements and the isotropic hardening of the yield surface. The stiffening effect is accomplished by the kinematics of the system. Two numerical 
models have been tested to simulate the hysteresis loop of the seismic device, which are schematically sketched in Figure 18. Basically a plastic spring is put in parallel with either contact elements which are themselves put in series with additional elastic springs or just one multilinear elastic spring. The plastic spring accounts for the yielding and the isotropic hardening effect, which is accomplished by choosing appropriate isotropic hardening parameters. The stiffening effect is modelled by the contact element and elastic spring series or the multilinear elastic spring. The more contact spring combinations or data points for the multilinear spring are used, the more smooth the post yielding curve will look like. Both systems are able to reproduce the same hysteresis curve shown in Figure 17. The fit between test and simulation seems to be acceptable taking into account the simplified modelling approach. Although both modelling approaches yield the same results, the second one proves to be more robust and computational efficient, mainly due to the lack of highly discontinuous contact elements. In general, no convergence issues could be observed for the second approach and runtime of simulations compared to the unretrofitted simulations just slightly increased.

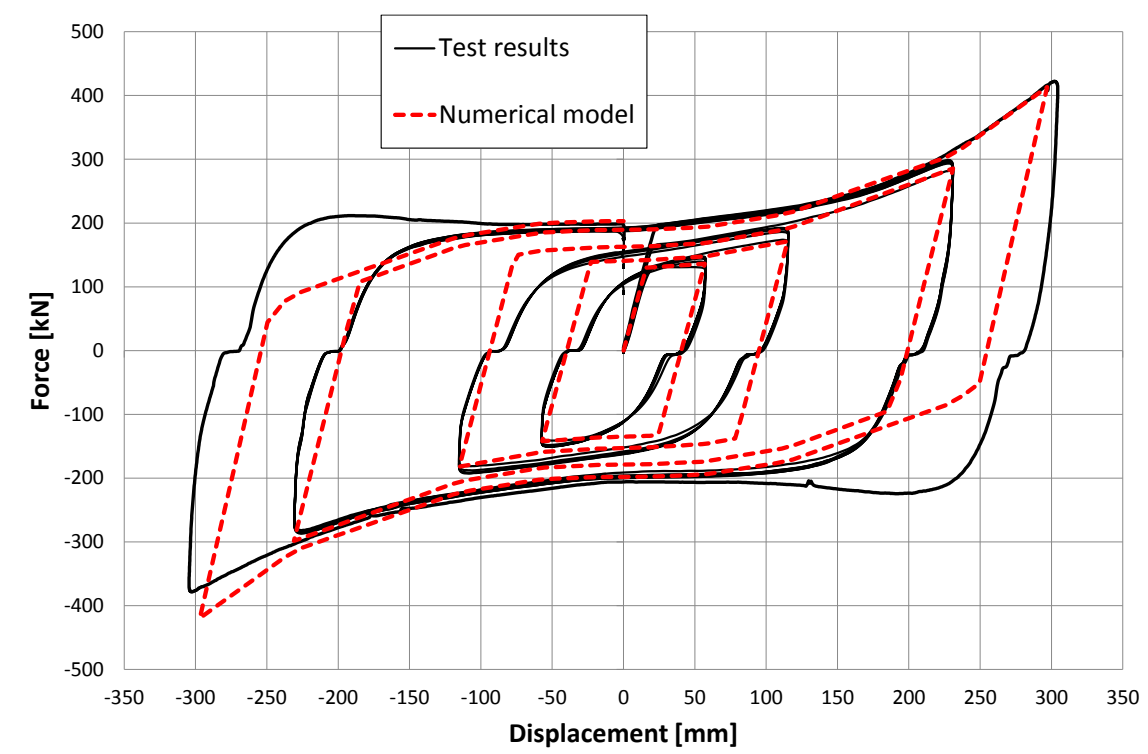

Figure 17: Typical force displacement hysteresis for torsional devices obtained by experimental tests (prototype with one default yielding core) [provided by MAURER SÖHNE] and results of numerical representation.

(a) Complex approach

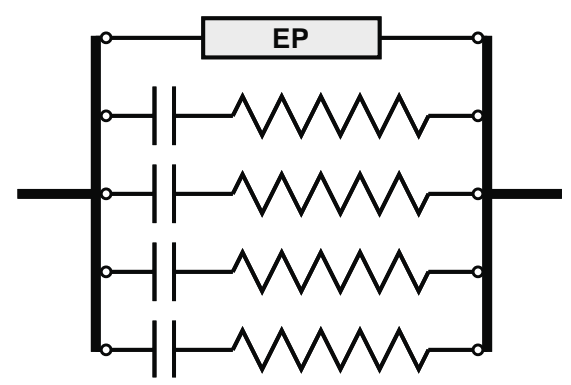

(b) Simplified approach

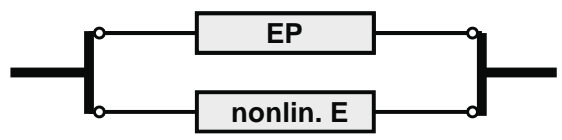

Figure 18: Rheological models to simulate the hysteresis of the torsional seismic device: approach with contact elements (a) and approach with multilinear elastic spring (b). 


\subsection{Retrofitting approach I: Energy dissipation in braced frames}

The torsional hysteretic device inserted between an added V-brace and the bottom of the superstructure has been modelled phenomenologically by inserting the rheological model discussed aboved at this spot. Figure 19 ashows a schematical sketch of this approach. The diagonals in the V-brace need to act both in tension as well as in compression. In order to prevent buckling the braces need to be sufficiently squat. In the simulations the flexibility of the Vbrace was assumed to be negligible compared to the flexibility of the device and thus was modelled as rigid.

The analysis conducted is considered as demand identification, i.e. how much torsional rods of the characteristic shown in Figure 17 are needed to improve the structural response significantly. The main purpose was to prevent the global sides-way collapse which happened in three of eleven earthquakes for the original structure. By an iterative procedure it was find out that in total 48 of torsional yield cores used for the whole structure would achieve this aim. Using thicker yielding cores the total amount would decrease accordingly. Number of cores per bay and number of bays need to be adapted to constructive and practicability boundary conditions. For example Figure 19 shows a device equipped with four yielding cores.
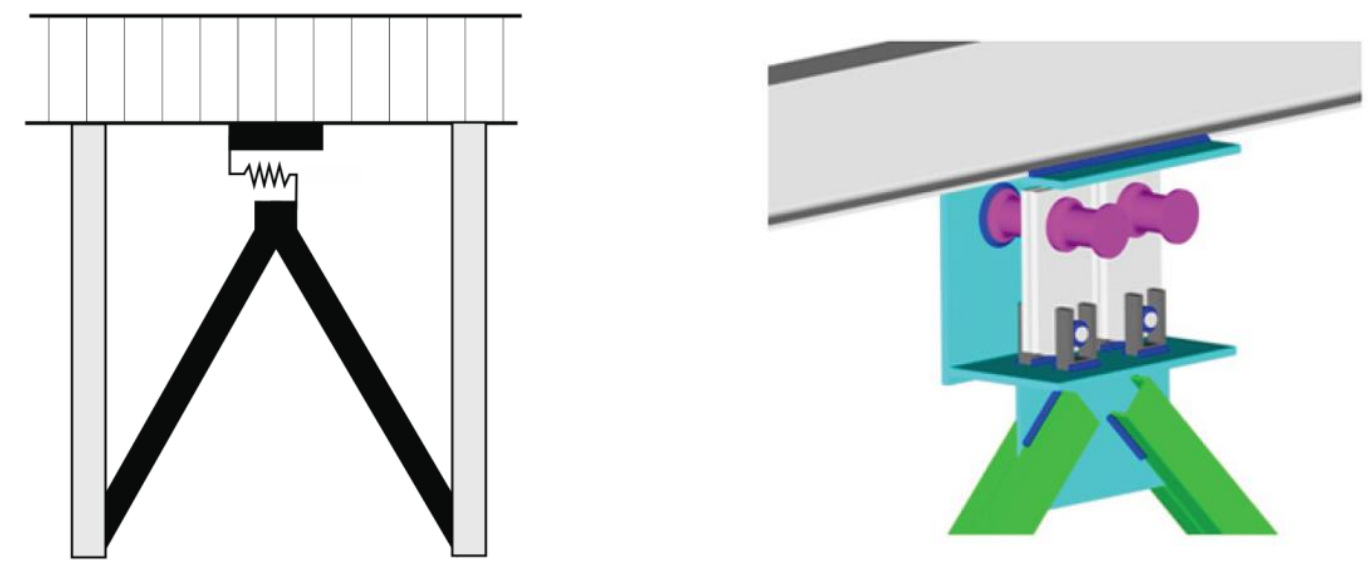

Figure 19: Schematical drawing of applied torsional hysteretic device into frame bay (left) and detail of prototype torsional device equipped with four yielding cores (right, [3]).

The applied retrofitting strategy adds additional amount of damping to the structure but also increases the stiffness of the longitudinal sway mode. Thus, this mode shifts to a higher acceleration value in the response spectrum which might cause an increase in demands. The modal periods of the structure after application of one of the discussed retrofitting strategies are listed in Table 8 while the mode numbering is in accordance to the mode shapes shown in Figure 6 to Figure 10.

\begin{tabular}{lccccc} 
& \multicolumn{4}{c}{ Modal period T [s] } \\
\cline { 2 - 5 } & As-built & \multicolumn{2}{c}{ Retrofit I } & \multicolumn{2}{c}{ Retrofit II } \\
\hline Mode 1 & 1.69 & 1.18 & $(70 \%)$ & 2.50 & $(150 \%)$ \\
Mode 2 & 1.24 & 1.24 & $(100 \%)$ & 2.09 & $(169 \%)$ \\
Mode 3 & 0.90 & 0.89 & $(100 \%)$ & 1.71 & $(190 \%)$ \\
Mode 4 & 0.72 & 0.72 & $(100 \%)$ & 0.89 & $(124 \%)$ \\
Mode 5 & 0.59 & 0.59 & $(100 \%)$ & 0.65 & $(110 \%)$ \\
\hline
\end{tabular}

Table 8: Modal periods after application of retrofitting. 
A comparison between the as-built structural performance and the retrofitted structure shows that with the determined amount of steel cores the failure mode of sides-way collapse could be completely excluded. Figure 20 shows the corresponding IDA curves. Whereby for the $\mathrm{H}-8$ peak displacement are still high - but without collapse taking place - the peak displacement for the other two earthquakes $\mathrm{H}-3$ and $\mathrm{H}-11$ are siginificantly reduced. Figure 21 shows a comparison for the no failure earthquakes. In general peak displacements are in most cases equal or lower, especially at the highest peak ground acceleration yielding a reduction of at least $20 \%$ can be obtained. The same holds true for the other earthquakes. Due to the period shift in some cases peak displacements may also be higher. The ratio of peak displacements of as-built to retrofitted behaviour is shown in Figure 22. Otherwise than the sides-way collapse failure mode, the other premature abortion reasons due to excessive brace deformations still occur. The behavior in transversal direction was essential the same, because the braced bays act in general just in longitudinal direction.

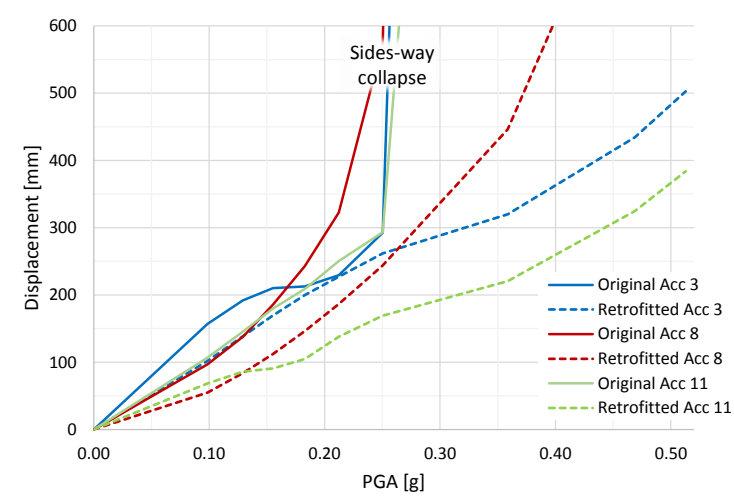

Figure 20: Comparison between as-built and retrofitted structure performance for sides-way collapse triggering earthquakes.

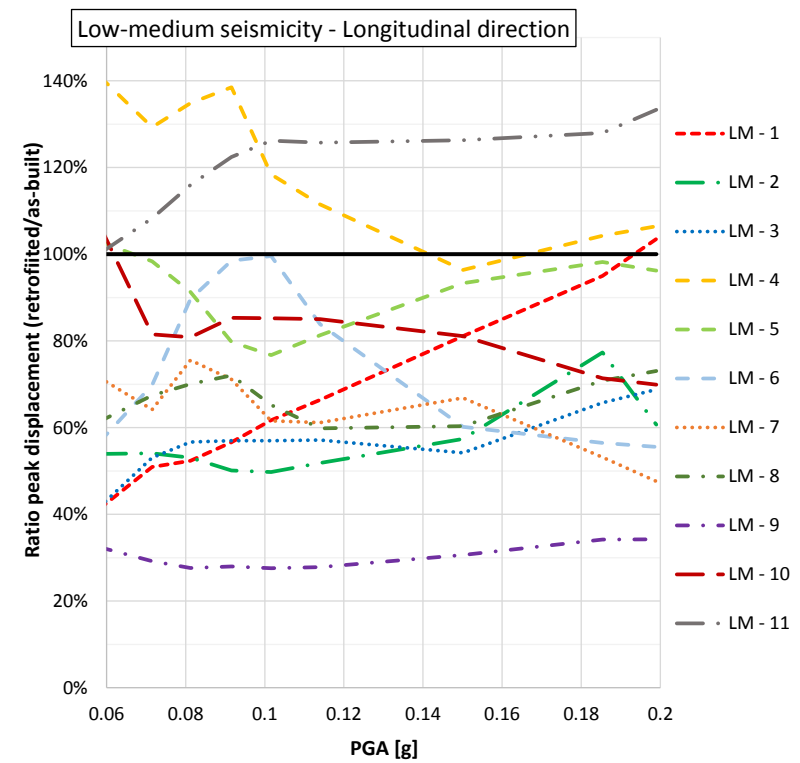

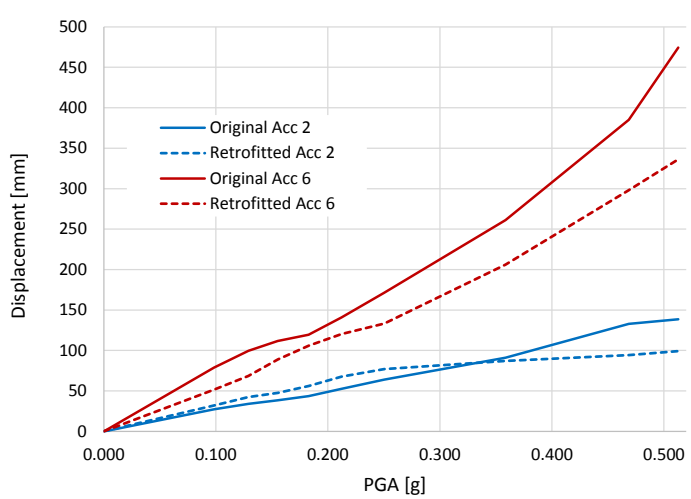

Figure 21: Comparison between as-built and retrofitted structure performance for no global failure earthquakes.

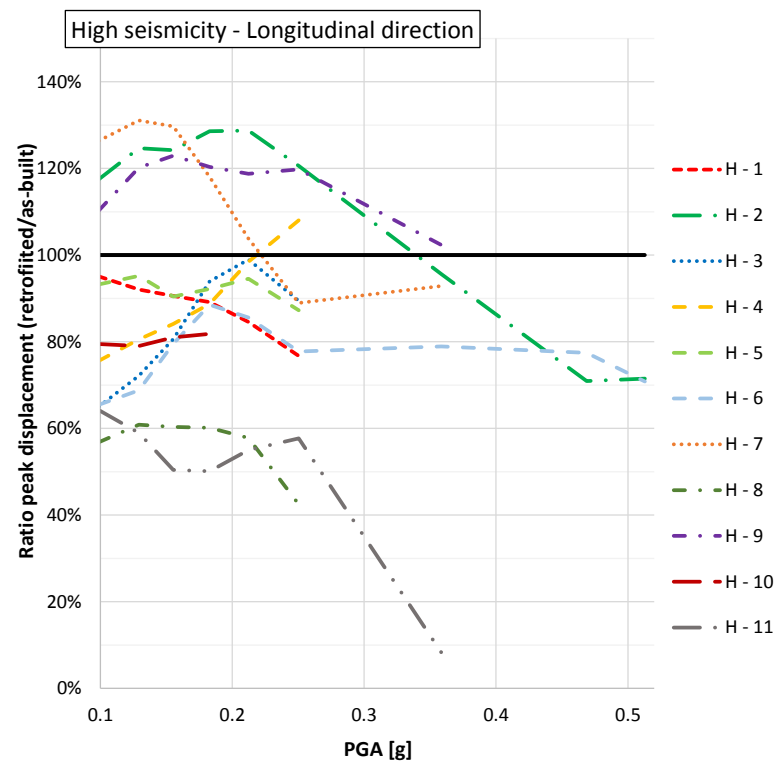

Figure 22: Ratio of as-built structure peak displacements in longitudinal X-direction to that ones of retrofitted structure for low to medium (left) and high (right) seismicity ground motion sets. 


\subsection{Retrofitting Approach II: Base isolation with torsional hysteretic dampers}

For the base isolation retrofitting strategy every column base has been separated from ground in both horizontal directions and the numerical model of the seismic device has been applied in both orthogonal directions. The hysteretic curve looks similar as that one shown in Figure 17 with the modification that the initial stiffness has been tuned in such a way to yield an increase in the period in longitudinal direction of $50 \%$, which yields a fundamental period of 2.5 seconds. The impact on the other modes is listed in Table 8.
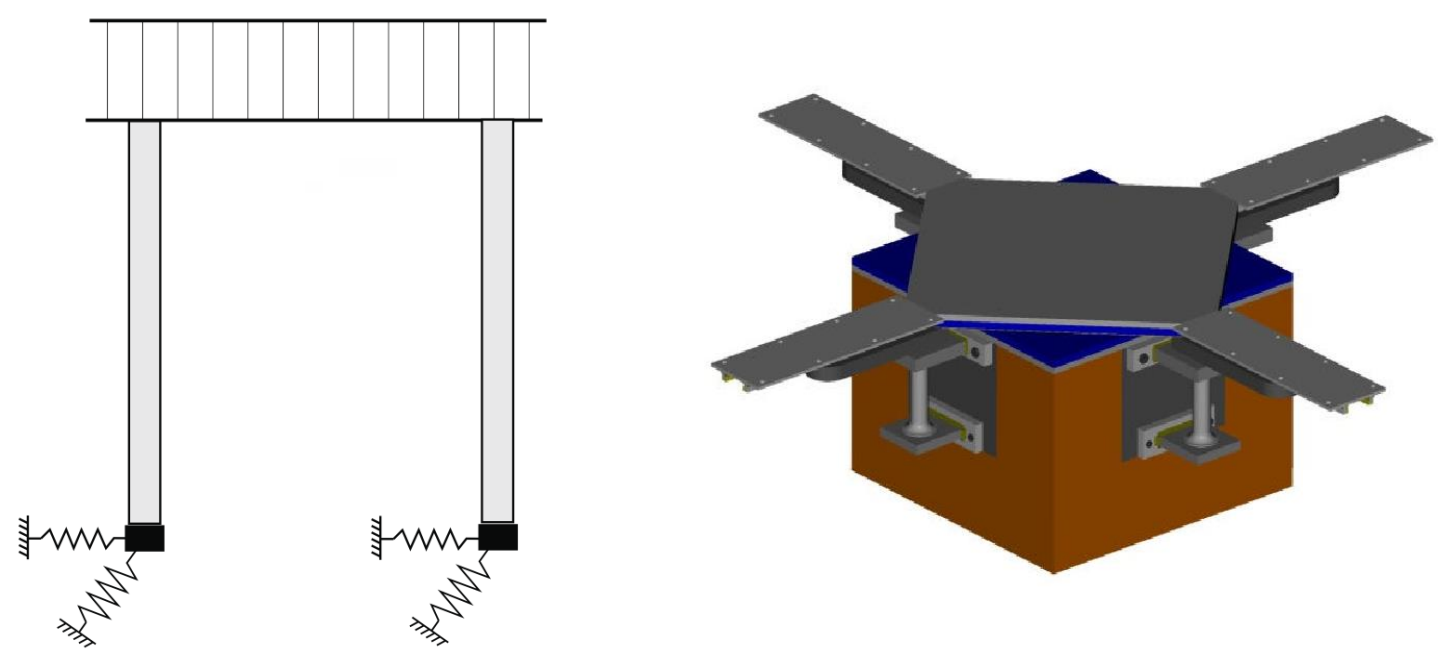

Figure 23: Schematical drawing of applied torsional hysteretic device as base isolation (left) and detail of multidirectional torsional hysteretic device (right, according to [4])

The retrofitted structure performed very well, as also for the high seismicity conditions no structural failure occurred. Figure 24 shows the relation between the peak displacements in the structure for both low to medium seismicity and high seismicity ground motions in longitudinal and transversal direction. For the retrofitted structure these are the displacements related to the top of the seismic device, which itself has to perform additional displacements. At higher peak ground accelerations for some high seismicity earthquakes the ratio was set to zero, in these cases when the as-built structure did not converge due to failure or excessive deformations. Otherwise the ratio is computed just for fully completed simulations. As can be seen for all of the ground motions deformation demand in the structure is efficiently decreased, for some even to $20 \%$ of its initial values.

The relative displacements inside the seismic device have been evaluated and are listed in Table 9. Demands have to be lower than capacity of the seismic device, otherwise the obtained results are not achievable in reality. For all of the low to medium seismicity earthquakes the displacements are well within the range of feasible displacements as can be compared to Figure 17. For some of the higher seismicity ground motions the demand on the seismic device is quite high for peak ground accelerations in the range of $0.25 \mathrm{~g}$ to $0.5 \mathrm{~g}$. However, up to a peak ground acceleration of $0.2 \mathrm{~g}$ also for all of the high seismicity ground motions the peak relative displacement is below $400 \mathrm{~mm}$, which is a well achievable capacity for this type of device. 

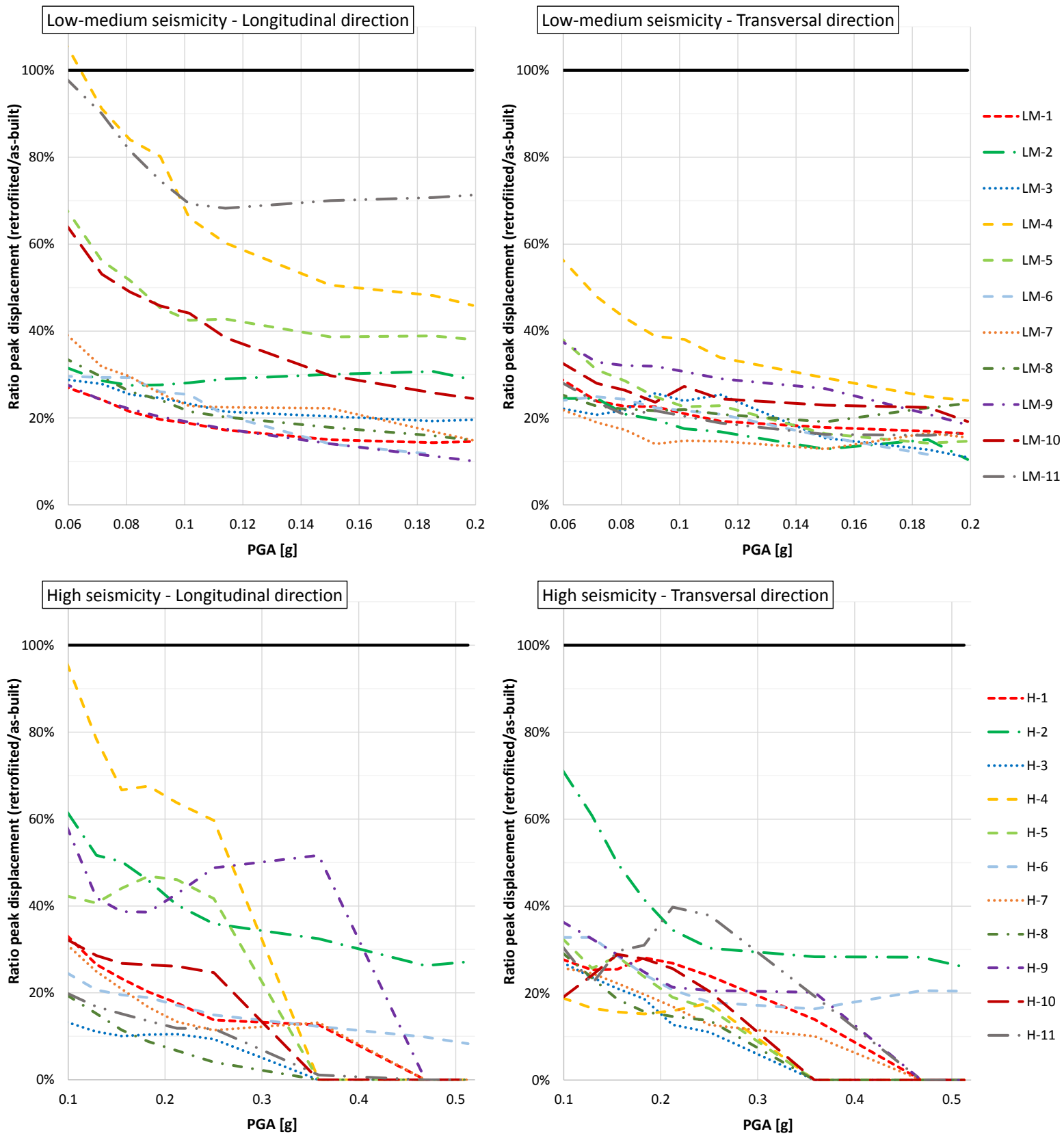

Figure 24: Ratio of structural relative displacements between as-built structure and retrofitted structure.

\begin{tabular}{ccccccccccccc}
\hline & \multicolumn{10}{c}{ Peak relative displacements in seismic device $\mathbf{u}_{\text {rel }}[\mathbf{m m}]$} \\
\hline LM- & $\mathbf{1}$ & $\mathbf{2}$ & $\mathbf{3}$ & $\mathbf{4}$ & $\mathbf{5}$ & $\mathbf{6}$ & $\mathbf{7}$ & $\mathbf{8}$ & $\mathbf{9}$ & $\mathbf{1 0}$ & $\mathbf{1 1}$ \\
\hline $\mathrm{X}$ & 91 & 150 & 141 & 53 & 95 & 143 & 150 & 216 & 205 & 109 & 49 \\
$\mathrm{Y}$ & 122 & 163 & 240 & 55 & 53 & 91 & 263 & 156 & 138 & 97 & 85 \\
\hline H- & $\mathbf{1}$ & $\mathbf{2}$ & $\mathbf{3}$ & $\mathbf{4}$ & $\mathbf{5}$ & $\mathbf{6}$ & $\mathbf{7}$ & $\mathbf{8}$ & $\mathbf{9}$ & $\mathbf{1 0}$ & $\mathbf{1 1}$ \\
\hline $\mathrm{X}$ & 364 & 163 & 431 & 128 & 345 & 512 & 665 & 1212 & 258 & 320 & 1282 \\
$\mathrm{Y}$ & 375 & 50 & 346 & 589 & 659 & 605 & 966 & 347 & 257 & 812 & 1016 \\
\hline
\end{tabular}

Table 9: Peak displacements in seismic device at highest scale factor for low to medium (LM) seismicity and high $(\mathrm{H})$ seismicity ground motions in longitudinal (X-) and transversal (Y-) direction. 


\section{CONCLUSIONS}

This paper has shown how a complex finite element model may be transferred into a simplified model to save computational effort and storage need by maintaining its most important dynamic properties. Such simplifications are needed to be able to handle the huge amount of seismic analyses which need to be conducted for the purpose of Incremental Dynamic Analyses.

With this simplified model Incremental Dynamic Analyses have been conducted to assess on the current performance. Further on, two retrofitting strategies have been applied in a generic way: Additional stiffness and energy dissipation with additional bracings equipped with seismic devices and a base isolation layer between column footings and ground. The simple modelling of the characteristic hysteresis by help of a rheological model has been discussed. While the first retrofitting strategy showed to be effective in preventing the global sides-way collapse failure mode, the second one moreover could drastically reduce displacement demands in all the earthquakes.

In these analyses the seismic devices have been applied in a generic way. In future the modelling strategies and used parameters for the seismic device will be investigated more deeply regarding practicability and economic issues. Moreover, further limit states have to be incorporated as solely the displacement or occurrence of global failure. The demand on the seismic device has to be compared in detail with its realistic capacity.

\section{ACKNOWLEDGMENTS}

The research leading to these results has received funding from the European Union's Research Fund for Coal and Steel (RFCS) research programme under grant agreement $\mathrm{n}^{\circ}$ RFSRCT-2013-00019, project "PROINDUSTRY - Seismic PROtection of INDUSTRial plants by enhanced steel based sYstems".

The test results of the prototype of the torsional seismic device have been provided by Maurer Söhne Engineering GmbH \& Co. KG, which is greatly acknowledged.

\section{REFERENCES}

[1] PROINDUSTRY, Seismic PROtection of INDUSTRial plants by enhanced steel based sYstems, Mid-Term Report, Grant Agreement Number: RFSR-CT-2013-00019, 2015.

[2] A. Salem Milani, TORSIONAL HYSTERETIC DAMPER FOR SEISMIC PROTECTION OF STRUCTURES, PhD Dissertation, Middle East Technical University, Turkey, May 2014.

[3] MULTI-DIRECTIONAL TORSIONAL HYSTERETIC DAMPER WITH RECENTERING CAPABILITY (MRSD) - A New Anti-Seismic Device for seismic protection of buildings and bridges, http://www.metu.edu.tr/announcement/profdr-murat-diclelis-seismic-damper, 10/10/2013.

[4] M. Dicleli; A. Salem Milani, MARTI and MRSD: Newly Developed Isolation-Damping Devices with Adaptive Hardening for Seismic Protection of Structures, International Journal of Civil, Environmental, Structural, Construction and Architectural Engineering, Vol: 9, No: 6, 2015. 